\title{
Event Refinement in State-Based Concurrent Systems
}

\author{
Jane Sinclair and Jim Woodcock \\ Programming Research Group, Oxford University Computing Laboratory, \\ Oxford, UK
}

Keywords: Refinement; Concurrency; State-based specification; Action systems

\begin{abstract}
Operations on action systems may be defined corresponding to CSP hiding and renaming. These are of particular use in describing the refinement between action systems in which the granularity of actions is altered. We derive a simplified expression for hiding sets of actions and present sufficient conditions for forwards simulation in which the concrete system uses hiding and renaming. Both of these reduce the complexity of proofs of refinement. We present a case study in specification and refinement using action systems which makes use of the operations and refinement rules previously defined.
\end{abstract}

\section{Introduction}

A state-based style of specification can provide a convenient and expressive notation for describing a computer-based system. This has been the experience of the collaborative project between Oxford University and IBM United Kingdom Laboratories Limited. On this project the specification language $Z$ [Spi89] has been used to specify part of IBM's Customer Information Control System (CICS ${ }^{1}$ ). A general overview of this work is given in [HoK91]. In some parts of CICS concurrency is encountered, and so the question arose as to how concurrency might be expressed whilst still maintaining a state-oriented approach.

In the work of Back and Kurki-Suonio [BKS83] action systems provide a state-based approach to concurrency. In an action system a collection of labelled

Correspondence and offprint requests to: Jane Sinclair, Programming Research Group, Oxford University Computing Laboratory, 7-11 Keble Road, Oxford OX1 3QD, UK.

1 A trademark of the IBM Corporation. 
actions share a state. An enabled action may be executed to change the state, resulting in a possibly altered set of enabled actions.

A good deal of research has been done on action systems and related topics. In Section 10 we consider some of these approaches and the relationship of our current work to existing formal methods in this field.

Here, we are concerned with building on work linking action systems with CSP [Hoa85]. In particular, Morgan [Mor90] has noted the attractiveness of combining both state-based and event-based styles of specification, allowing the strengths of each to be exploited where appropriate. In [Mor90] the work on action systems is extended to provide a direct link between action systems and CSP. [WoM90] furthers this work by expressing CSP traces, failures and divergences as weakest precondition formulae over action systems. An advantage of this approach is its simplified treatment of divergence.

In [WoM90] definitions of both forwards and backwards simulations are derived for action systems and are shown to be sound with respect to failuresdivergence refinement in CSP. To establish forwards simulation using the definition of [WoM90] it must be shown that:

- initialisation of the concrete system corresponds to initialisation of the abstract system

- each concrete action corresponds to an abstract action

- each concrete action is enabled whenever its abstract counterpart is enabled

(these requirements are stated formally in section 6). One of the benefits of this result is to maintain the link with CSP through refinement whilst providing refinement rules for each action, thus obviating the need for tedious proofs over all possible traces.

The application we are considering features some operations which, for ease of specification, are represented by a single action, but which cannot be performed atomically in the target language. Therefore we allow the concrete system to have added housekeeping operations, referred to as 'silent' actions. We impose the constraint that repetition of silent actions must be bounded. However, a system with added silent actions would not be a refinement in the sense of [WoM90] since each action of the concrete system, even the silent ones, would be expected to be a refinement of one abstract operation. To overcome this we make use of two operations on action systems, namely hiding and renaming, which are defined to correspond to their CSP equivalents. The silent nature of an operation can be reflected by hiding it within the action system. A hidden action no longer appears as a separate action within the system but may be performed repeatedly both before and after each of the remaining actions. A formal definition is given in Section 4.

The need for renaming arises from another alteration in the granularity of actions. The concrete system introduces a set of users and splits single actions of the specification into a set of user-indexed actions. Renaming can be used to provide a correspondence between a set of concrete actions and a single abstract action.

It can then be shown, using the result derived in [WoM90], that the resulting system with silent actions hidden and sets of actions renamed is indeed a refinement of the abstract system. Calculating this resulting system and then proving the refinement is unnecessarily tedious. We present sufficient conditions for showing refinement in one step with much simplified calculations.

This paper continues the work of [Mor90], [WoM90] in bringing together the respective state-based and event-based approaches of action systems and CSP, 
using CSP-type hiding and renaming operators to provide greater expressive power. Proof of forwards simulation in which the concrete system uses hiding and renaming is facilitated by the derivation of a simplified set of sufficient conditions. These ideas are drawn together in the presentation of a case study based on an example arising from our work with IBM.

\section{Action Systems and Guarded Commands}

Action systems, defined in [BKS83], provide a state-based approach to specifying concurrent systems. An action system, $\mathscr{A}$, consists of a state, $x$, an initialisation, $\mathscr{A}_{\text {init }}$, and a set of labelled actions, $\left\{\mathscr{A}_{i} \mid i \in L\right\}$, where $L$ is an alphabet of labels. Each action, $\mathscr{A}_{i}$ consists of a guard, $g_{i}$, which is a predicate, and a command, $A_{i}$ which is a program fragment from Dijkstra's guarded command language [Dij76]. An action is said to be enabled when its guard evaluates to true. The initialisation is a command. We use the notation:

$$
\mathscr{A} \widehat{=}\left(\begin{array}{l}
\operatorname{var} x: X \\
\text { initially } \mathscr{A}_{\text {init }} \\
\vdots \\
i: g_{i} \rightarrow A_{i} \\
\vdots
\end{array}\right)
$$

Execution of an action system starts with execution of the initialisation. Repeatedly, an enabled action is selected and executed. If no action is enabled the system is deadlocked. If an executed action aborts, the system is said to diverge.

Guarded commands may be given a weakest precondition semantics as in [Mor90]:

Definition 1. For guard $g$, command $A$, and postcondition $\alpha$

$$
w p(g \rightarrow A, \alpha) \widehat{=} g \Rightarrow w p(A, \alpha) .
$$

घ

All commands allowed by [Dij76] obey the Law of the Excluded Miracle (that is, $w p(A, f a l s e)=$ false for any command, $A$ ). However, this is not necessarily the case for a guarded command. For example, $w p($ false $\rightarrow$ skip, false $)=$ true. Throughout this paper we will maintain the distinction between a command and a guarded command. As stated above, a command is simply a program fragment from [Dij76], and we can make use of the many well-known properties of commands. In general, we will use letters $P, Q, R$ to represent arbitrary guarded commands and $A, B, C$ for arbitrary commands.

Guarded commands do obey some of the same laws as commands. For example, we can establish monotonicity as follows. If $\alpha \Rightarrow \beta$ then by monotonicity of commands, $w p(A, \alpha) \Rightarrow w p(A, \beta)$. Hence

$$
\begin{aligned}
& w p(g \rightarrow A, \alpha) \\
& =g \Rightarrow w p(A, \alpha) \\
& \Rightarrow g \Rightarrow w p(A, \beta) \\
& =w p(g \rightarrow A, \beta)
\end{aligned}
$$
[transitivity of $\Rightarrow$ ] [defn. 1] 
We will make use of the conjugate weakest precondition which characterises the states from which a command, $A$ might possibly establish the postcondition, $\alpha$ :

\section{Definition 2.}

$\overline{w p}(A, \alpha) \bumpeq \neg w p(A, \neg \alpha)$.

घ

As noted in [WoM90], this interpretation sees an aborting command as possibly establishing anything.

Definition 2 can be extended to guarded commands. Calculation then reveals that

$\overline{w p}(g \rightarrow A$, true $)=g$

So, for a guarded command, $P, \overline{w p}(P$, true $)$ characterises the states in which $P$ is enabled.

Some additional notation will be useful for defining hiding. We first introduce a choice operator between guarded commands.

\section{Definition 3.}

$$
(g \rightarrow A \backslash h \rightarrow B) \widehat{=}(g \vee h \rightarrow \text { if } g \rightarrow A \rrbracket h \rightarrow B \text { fi })
$$

占

The weakest precondition may be calculated using Definition 1:

Proposition 1. For any postcondition, $\alpha$

$$
w p(P \backslash Q, \alpha)=w p(P, \alpha) \wedge w p(Q, \alpha)
$$

and

$$
\overline{w p}(P \mid Q, \alpha)=\overline{w p}(P, \alpha) \vee \overline{w p}(Q, \alpha) .
$$

七

Amongst the properties which follow from the above definitions we will make use of:

Proposition 2. For guarded commands $P, Q$ and $R$

$$
\begin{aligned}
& P ;(Q \mid R)=(P ; Q) \mid(P ; R) \\
& (P \mid Q) ; R=(P ; R) \mid(Q ; R) \\
& (P \mid Q)|R=P|(Q \mid R) \\
& P|Q=Q| P
\end{aligned}
$$

与

The associativity result, [iii], allows us to omit brackets from multiple choices. We will write

$$
\prod_{i \in\{1 \ldots n\}} a c t_{i}
$$


instead of

$$
a_{1}\left|\operatorname{act}_{2}\right| \cdots \mid a c t_{n} .
$$

Using this notation we record one further property:

Proposition 3. If $S$ and $T$ are two indexing sets then

$$
\varliminf_{i \in S \cup T)} a c t_{i}=\left(\prod_{i \in S} a c t_{i}\right) \backslash\left(\prod_{i \in T} a c t_{i}\right)
$$

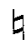

As an aid to simplifying expressions later in the paper we identify the "impossible" action - that is one which always establishes the postcondition false.

Definition 4. $P$ is impossible iff $w p(P$, false $)=$ true 4

This is closely linked to the definition of a trace given in [WoM90] - a trace, tr, being a finite sequence of actions such that $\overline{w p}(t r, t r u e)$ is true initially. The empty trace, $\langle$, is equivalent to skip. Since $w p(P$, false $)=\neg \overline{w p}(P$, true $)$, Definition 4 simply says that $P$ is not a trace in this sense. In fact, $P$ acts like the CSP process STOP which is a unit for general choice. The following lemmas confirm this behaviour and will prove useful for eliminating impossible branches from expressions:

Lemma 1. If $P$ is impossible, so is $P ; Q$.

If in addition $w p(Q$, true $)=$ true then $Q ; P$ is impossible too.

Proof. Since by monotonicity, $w p(P, f a l s e) \Rightarrow w p(P, \alpha)$ for any postcondition, $\alpha$ :

$$
\begin{aligned}
& w p(P ; Q, \text { false }) \\
& =w p(P, w p(Q, \text { false })) \\
& =\text { true }
\end{aligned}
$$

Also, with the further assumption $w p(Q$, true $)=$ true :

$$
\begin{aligned}
& w p(Q ; P, \text { false }) \\
& =w p(Q, w p(P, \text { false })) \\
& =w p(Q, \text { true }) \\
& =\text { true }
\end{aligned}
$$

曰

Lemma 2. If $P$ is impossible, then $P \mid Q=Q$.

Proof. For any postcondition $\alpha$

$$
\begin{aligned}
& w p(P \mid Q, \alpha) \\
& =w p(P, \alpha) \wedge w p(Q, \alpha) \\
& =\operatorname{true} \wedge w p(Q, \alpha) \\
& =w p(Q, \alpha)
\end{aligned}
$$

[Proposition 1] [P impossible] [prop. calc.] 
Lemma 3. Let $P$ be such that $w p(P, \neg h)=$ true and $Q \widehat{=} h \rightarrow B$ then $P ; Q$ is impossible.

Proof.

$$
\begin{aligned}
& w p(P ; Q, \text { false }) \\
& =w p(P, w p(Q, f a l s e)) \\
& =w p(P, h \Rightarrow w p(B, \text { false })) \\
& =w p(P, \neg h) \\
& =\text { true }
\end{aligned}
$$
[Definition 1] [Ex. miracle for $B$ ] [Assumption]

\section{An Iterative Command}

When an action is hidden it may be performed repeatedly before and after each of the remaining commands. To reflect this we make use of the iterative command, it $P$ ti , defined in [BuM91]. This allows repetition of $P$ with the possibility of terminating with skip at each iteration. The definition makes use of the choice operator for guarded commands and the operator, $\mu$, which gives the least fixed point with respect to the refinement ordering.

\section{Definition 5.}

$$
\text { it } P \text { ti } \widehat{=}(\mu X \bullet \text { skip } \mid P ; X)
$$

Ł

Unwinding the definition for iteration gives:

$$
\begin{aligned}
& \text { it } P \mathbf{t i} \\
& =\operatorname{skip} \mid P ;(\text { it } P \text { ti }) \\
& =\operatorname{skip} \mid P ;(\operatorname{skip} \mid P ;(\text { it } P \text { ti })) \\
& =\operatorname{skip}(P ; \operatorname{skip}) \mid(P ; P ;(\text { it } P \text { ti })) \\
& =\operatorname{skip} P \mid(P ; P ;(\text { it } P \text { ti })) \\
& =\operatorname{skip}|P|(P ; P) \backslash(P ; P ; P ;(\text { it } P \text { ti }))
\end{aligned}
$$

[Definition 5] [Expanding definition] [Proposition 2] [defn. of skip] [Unwinding further]

If for a particular $P$ it can be shown that iterating $P k$ times is impossible then the lemmas of the previous section can be used to simplify the above expression.

Following this unwinding and using Proposition 1 it can be seen informally that the conjugate weakest precondition for it $P$ ti to possibly establish some postcondition, $\alpha$, is

$$
\overline{w p}(s k i p, \alpha) \vee \overline{w p}(P, \alpha) \vee \overline{w p}(P ; P, \alpha) \vee \ldots
$$

The correspondence between action systems and CSP reported in [Mor90] and [WoM90] is based on expressing CSP failures and divergences as weakest precondition formulae over action systems. As noted in [BuM91], this model 
does not treat unbounded nondeterminism. [BuM91] demonstrates how hiding in an unboundedly nondeterministic action system destroys the link with CSP. Also, if the set of actions to be hidden may give rise to possibly infinite iteration then unbounded nondeterminism may be introduced into a boundedly nondeterministic system. To deal with this, [BuM91] extends the CSP - action system correspondence using the infinite traces model of CSP [Ros88].

Our consideration is at present restricted to action systems in which nondeterminism is bounded. We impose the additional constraint that iteration of sets of actions to be hidden must also be bounded. So for any hidden set of actions, $\mathscr{H}$, there will be a bound function, $b f_{\mathscr{H}}$ which for any allowed state gives the maximum number of iterations of actions of $\mathscr{H}$ to ensure termination. Thus the refinement rules of [WoM90] are sufficient for the purposes of this paper. However, it would be possible to continue Butler's work in the infinite traces model to provide refinement rules for action systems allowing unbounded nondeterminism.

The above discussion motivates the following definition which follows from the more general infinite traces model derived in [BuM91].

Definition 6. For a set of actions, $\mathscr{H}$, let $\mathscr{H}^{*}$ be the set of traces formed by taking finite sequential compositions of actions in $\mathscr{H}$. Then, provided iteration in $\mathscr{H}$ is bounded, for any postcondition $\alpha$

$$
\overline{w p}\left(\text { it }\left.\right|_{h \in \mathscr{H}^{*}} h \mathbf{t i}, \alpha\right)=\exists \operatorname{tr}: \mathscr{H}^{*} \bullet \overline{w p}(t r, \alpha)
$$

b

Any iteration may establish the postcondition true. This is because iteration may choose to skip immediately.

Lemma 4. For any set of actions $\mathscr{H}$

$$
\overline{w p}\left(\text { it }\left.\right|_{h \in \mathscr{H}} h \mathbf{t i}, \text { true }\right)=\text { true }
$$

Proof. The empty trace, \langle\rangle , is a member of $\mathscr{H}^{*}$ for any $\mathscr{H}$ and

$$
\begin{aligned}
& \overline{w p}(\langle\rangle, \text { true }) \\
& =\overline{w p}(\text { skip, true }) \\
& =\text { true }
\end{aligned}
$$

[wp. calc.]

与

\section{Hiding for an Action System}

Suppose $\mathscr{A}$ is an action system with initialisation $\mathscr{A}_{\text {init }}$ and labelled actions $\left\{\mathscr{A}_{i} \mid i \in L\right\}$. For $M \subseteq L$ the effect on $\mathscr{A}$ of hiding all the actions labelled by $M$ is to remove those actions from the system. The remaining actions are modified by allowing iterations of actions from $M$ both before and after each one. Such iteration is also allowed after the initialisation. We write $\mathscr{A} \backslash M$ to denote the system resulting from hiding $M$ in $\mathscr{A}$. 
Definition 7. Let

$$
I T_{M}=\text { it } \prod_{i \in M} \mathscr{A}_{i} \mathbf{i}
$$

then

$$
(\mathscr{A} \backslash M)_{\text {init }}=\mathscr{A}_{\text {init }} ; I T_{M}
$$

and for each $j \in(L-M)$

$$
(\mathscr{A} \backslash M)_{j}=I T_{M} ; \mathscr{A}_{j} ; I T_{M}
$$

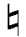

As mentioned above, if each action of $\mathscr{A}$ is boundedly nondeterministic and $I T_{M}$ is bounded then this definition for an action system corresponds to CSP hiding in the failures-divergences model. Action system hiding therefore enjoys the familiar properties of CSP hiding.

It may sometimes be useful to make explicit the guards of the actions in the new system. To calculate the guard of an action we follow [Mor90] and use the fact that for a command, $A$,

$$
g=\overline{w p}(g \rightarrow A, \text { true })
$$

Lemma 5. For any iteration, $T$, and action, $g \rightarrow A$

$$
\overline{w p}((T ; g \rightarrow A ; T), \text { true })=\overline{w p}(T, g)
$$

Proof For any iteration, $T$ :

$$
\begin{aligned}
& \overline{w p}((T ; g \rightarrow A ; T), \text { true }) \\
& =\neg w p(T, w p(g \rightarrow A, w p(T, \text { false }))) \\
& =\neg w p(T, g \Rightarrow w p(A, w p(T, \text { false }))) \\
& =\overline{w p}(T, g \wedge \overline{w p}(A, \overline{w p}(T, \text { true }))) \\
& =\overline{w p}(T, g \wedge \overline{w p}(A, \text { true })) \\
& =\overline{w p}(T, g \wedge \text { true }) \\
& =\overline{w p}(T, g)
\end{aligned}
$$

\section{Renaming for an Action System}

Suppose $\mathscr{A}$ is an action system and $f$ is a total function which maps labels of $\mathscr{A}$ $\left(L\right.$, say) surjectively to possibly different labels, $L^{\prime}$ :

$$
f: L \longrightarrow L^{\prime}
$$

Following the example of CSP renaming we wish to rename $\mathscr{A}$ using the relabelling function, $f$. There is no requirement for $f$ to be injective, so applying $f$ to the labels of $\mathscr{A}$ could result in several actions sharing a single label. In such cases the actions for that label are brought together using the choice operator. The resulting system is defined as follows:

Definition 8. For action system $\mathscr{B}$ formed by renaming $\mathscr{A}$ with $f$ :

$$
\mathscr{B}_{\text {init }} \widehat{=} \mathscr{A}_{\text {init }}
$$


and for each $l^{\prime}: L^{\prime}$ :

$$
\mathscr{B}_{l^{\prime}} \widehat{=} \mathscr{A}_{l} \quad \text { for } l \in\left\{l: L \mid f l=l^{\prime}\right\}
$$

\section{Refinement for an Action System}

We give the definition of forwards simulation between action systems derived in [WoM90].

Consider an abstract system $\mathscr{A}$ and concrete system $\mathscr{C}$ with states $a$ and $c$ respectively. A relation $R$ between $\mathscr{A}$ and $\mathscr{C}$ is a forwards simulation, written $R: \mathscr{A} \sqsubseteq \mathscr{C}$, if for any $\beta$ not containing abstract variables $a$ it satisfies the following three rules:

- The initialisation rule

$$
\left(\exists c \bullet \overline{w p}\left(\mathscr{C}_{\text {init }}, \beta\right)\right) \Rightarrow\left(\exists a \bullet \overline{w p}\left(\mathscr{A}_{\text {init }},(\exists c \bullet R \wedge \beta)\right)\right)
$$

Any state established by the concrete initialisation must correspond to one allowed by the abstract initialisation.

- The correctness rule

For each $a c t_{i}$

$$
\left(\exists c \bullet R \wedge \overline{w p}\left(\mathscr{C}_{a c t_{i}}, \beta\right)\right) \Rightarrow \overline{w p}\left(\mathscr{A}_{a c t_{i}},(\exists c \bullet R \wedge \beta)\right)
$$

Starting from abstract and concrete states related by $R$, any state reached by a concrete action must correspond to one reached by the matching abstract action.

- The applicability rule

For each $a c t_{i}$

$$
\left(\exists a \cdot R \wedge g_{\mathscr{A}_{\text {act }_{i}}}\right) \Rightarrow g_{\mathscr{C}_{\text {act }}}
$$

If an abstract action is enabled then the corresponding concrete action must also be enabled.

\section{Case Study}

The abstract action system, $\mathscr{P}$, describes a simple situation in which an integer variable, $u$ may be incremented or decremented.

$$
\begin{aligned}
& \operatorname{var} u: \mathbb{Z} \bullet \\
& \text { initially } u:=0 \\
& \text { inc }: \text { true } \rightarrow u:=u+1 \\
& \text { dec }: \text { true } \rightarrow u:=u-1
\end{aligned}
$$

The proposed implementation, $\mathscr{I}$, includes a set of $n$ users and additional operations for locking and unlocking. This would be necessary in the case of actions with commands for which no atomic equivalent is available in the target language. The idea is that a user is made to lock the resource before performing an action corresponding to one of the abstract ones, and release it by unlocking afterwards. We introduce the type Owner consisting of the users $(1 \ldots n)$ and an 
additional value, null, to indicate when no user owns the lock. To ensure the correct succession of events the program control variable, $p c$, is used.

var $v: \mathbb{Z}, p c:\{0,1,2\}$,owner : Owner and $p c=0 \Leftrightarrow$ owner $=$ null $\bullet$

initially $v:=0 ; p c:=0$; owner $:=$ null

lock $_{i}: p c=0 \wedge$ owner $=$ null $\rightarrow p c:=1 ;$ owner $:=i$

cinc $_{i}: p c=1 \wedge$ owner $=i \rightarrow p c:=2 ; v:=v+1$

cdec $_{i}: p c=1 \wedge$ owner $=i \rightarrow p c:=2 ; v:=v-1$

unlock $_{i}: p c=2 \wedge$ owner $=i \rightarrow p c:=0$; owner $:=$ null

This action system includes the invariant, $p c=0 \Leftrightarrow$ owner $=$ null which must be established by the initialisation and, for each action, $a c t_{i}$, if $a c t_{i}$ is enabled and the invariant holds then termination of $a c t_{i}$ must re-establish the invariant. A routine calculation shows that $p c=0 \Leftrightarrow$ owner $=$ null is indeed an invariant for $\mathscr{I}$. For user $i$ to lock the resource, the owner must initially be null and is then set to $i$. The control variable $p c$ is set to 1 which ensures that only an increment or decrement action is now enabled. This prevents possible divergence caused by repetition of lock and/or unlock. After $v$ has been updated $p c$ is given the value 2 thus enabling unlock which sets $p c$ back to 0 and the owner to null. Thus the simple abstract operation of a user incrementing the variable may now be represented by the sequence $\operatorname{lock}_{i} ; \operatorname{cinc}_{i} ;$ unlock $k_{i}$ and similarly for dec, destroying the atomicity of actions. We wish to show that system $\mathscr{I}$ refines $\mathscr{P}$ in some sense, but it is not the case that each concrete action refines exactly one abstract action. Each of the silent lock , unlock $_{i}$ operations must be hidden in the concrete system. We will use $\mathscr{H}$ to denote the set of all these silent actions.

There is a further complication. The concrete system introduces the set of users and separate operations, $c i n c_{i}, c d e c_{i}$, for each user, $i$. The $n \operatorname{cinc}_{i}$ operations must be made to correspond to the one abstract inc operation, and similarly for dec. To achieve this we will make use of the renaming operation defined above which has the effect of combining the $\operatorname{cinc}_{i}$ and $c d e c_{i}$ operations in the concrete system. We will show that for a suitable renaming function, rename:

\section{$\mathscr{S} \sqsubseteq$ rename $(\mathscr{I} \backslash \mathscr{H})$}

\subsection{Hiding Silent Actions}

The first step is to calculate $\mathscr{I} \backslash \mathscr{H}$ where $\mathscr{H}$ contains all the lock $k_{i}$ and unlock $_{i}$. If we define:

$$
T \cong \text { it }\left.\right|_{h \in \mathscr{H}} \mathscr{I}_{h} \mathbf{t i}
$$

then using Proposition 3, for each $k$ with $1 \leq k \leq n$ the actions after hiding are:

$$
\begin{aligned}
& (\mathscr{I} \backslash \mathscr{H})_{\text {cinc }_{k}}=T ;(p c=1 \wedge \text { owner }=k \rightarrow p c:=2 ; v:=v+1) ; T \\
& (\mathscr{I} \backslash \mathscr{H})_{\text {ddec }_{k}}=T ;(p c=1 \wedge \text { owner }=k \rightarrow p c:=2 ; v:=v-1) ; T
\end{aligned}
$$

The iteration expression for $T$ can be simplified by first expanding using the "unwinding" given above:

$$
\text { it } P \text { ti }=\operatorname{skip}|P|(P ; P) \mid(P ; P ; P ;(\text { it } P \text { ti }))
$$

and then removing impossible branches. Using this approach and representing the locking and unlocking actions in $\mathscr{I}$ by their labels we show that: 


\section{Lemma 6.}

$$
T=\operatorname{skip}\left|\left(\left.\right|_{i} \operatorname{lock}_{i}\right)\right|\left(\prod_{i} \text { unlock }_{i}\right) \mid\left(\prod_{i, j}\left(\text { unlock }_{i} ; \operatorname{lock}_{j}\right)\right) \text { for } 1 \leq i, j \leq n
$$

Proof.

$$
\begin{aligned}
& P ; P \\
& =\left(\left(\left.\right|_{i} \operatorname{lock}_{i}\right) \mid\left(\left.\right|_{i} \text { unlock }_{i}\right)\right) ;\left(\left(\left.\right|_{i} \operatorname{lock}_{i}\right) \backslash\left(\left.\right|_{i} \text { unlock }_{i}\right)\right) \quad \text { [By definition] } \\
& =\left(\left(\left.\right|_{i} \operatorname{lock}_{i}\right) ;\left(\left.\right|_{i} \operatorname{lock}_{i}\right)\right) \backslash\left(\left(\left.\right|_{i} \text { unlock }_{i}\right) ;\left(\left.\right|_{i} \text { lock }_{i}\right)\right) \mid \\
& \quad\left(\left(\left.\right|_{i} \operatorname{lock}_{i}\right) ;\left(\left.\right|_{i} \text { unlock }_{i}\right)\right) \mid\left(\left(\left.\right|_{i} \text { unlock }_{i}\right) ;\left(\left.\right|_{i} \text { unlock }_{i}\right)\right) \text { [Proposition 2] }
\end{aligned}
$$

It is possible to show that the first, third and fourth branches of this expression are impossible for any $i, j:\{1, \ldots, n\}$. For example

$$
\begin{aligned}
& \left(\prod_{i} \operatorname{lock}_{i}\right) ;\left(\prod_{i} \operatorname{lock}_{i}\right) \\
& =\left(\operatorname{lock}_{1} \backslash \ldots \mid \operatorname{lock}_{n}\right) ;\left(\operatorname{lock}_{1} \backslash \ldots \mid \text { lock }_{n}\right) \\
& =\prod_{i, j}\left(\operatorname{lock}_{i} ; \operatorname{lock}_{j}\right)
\end{aligned}
$$

and since

$$
\begin{aligned}
& \text { wp }(\text { lock }, \neg(p c=0 \wedge \text { owner }=\text { null })) \\
& \begin{aligned}
&=(p c=0\wedge \text { owner }=\text { null }) \\
& \quad \Rightarrow w p(p c:=1 ; \text { owner }:=i, \neg(p c=0 \wedge \text { owner }=\text { null }))
\end{aligned} \\
& =(p c=0 \wedge \text { owner }=\text { null }) \Rightarrow \neg(1=0 \wedge i=\text { null }) \\
& =\text { true }
\end{aligned}
$$$$
\text { [prop. calc.] }
$$

by Lemma 3, lock $_{i} ; \operatorname{lock}_{j}$ is impossible for all $i, j:(1 \ldots n)$, and hence by Lemma 2 $\left(\right.$ lock $\left._{i} ; \operatorname{lock}_{j}\right)$ is impossible. A similar argument holds for the third and fourth branches, and so again by Lemma 2 :

$$
\begin{aligned}
& P ; P \\
& =\left(\left(\prod_{i} \text { unlock }_{i}\right) ;\left(\prod_{i} \text { lock }_{i}\right)\right) \\
& =\prod_{i, j}\left(\text { unlock }_{i} ; \text { lock }_{j}\right)
\end{aligned}
$$

Now we consider the next level of unwinding:

$$
\begin{aligned}
& P ; P ; P \\
& =\left(\left(\prod_{i} \operatorname{lock}_{i}\right) \backslash\left(\prod_{i} \text { unlock }_{i}\right)\right) ;\left(\prod_{i, j}\left(\text { unlock }_{i} ; \text { lock }_{j}\right)\right) \\
& =\left(\prod_{i, j, k}\left(\text { lock }_{i} ; \text { unlock }_{j} ; \text { lock }_{k}\right)\right) \backslash\left(\prod_{i, j, k}\left(\text { unlock }_{i} ; \text { unlock }_{j} ; \text { lock }_{k}\right)\right)
\end{aligned}
$$


Both branches of this are impossible, so from expression (1) using Lemmas 1 and 2 we have:

$$
T=\operatorname{skip}\left|\left(\left.\right|_{i} \operatorname{lock}_{i}\right)\right|\left(\left.\right|_{i} \text { unlock }_{i}\right) \mid\left(\left.\right|_{i, j}\left(\text { unlock }_{i} ; \text { lock }_{j}\right)\right)
$$

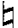

This result shows that repetition within $T$ is certainly bounded since no more than two actions may be performed consecutively.

The remaining actions may now be simplified. Firstly, since

$$
\begin{aligned}
& w p\left(l_{\text {lock }}, \neg(p c=1 \wedge \text { owner }=k)\right. \\
& =(p c=0 \wedge \text { owner }=\text { null }) \Rightarrow \neg(1=1 \wedge i=k) \\
& =(p c=0 \wedge \text { owner }=\text { null }) \Rightarrow i \neq k
\end{aligned}
$$

by Lemma 3 , if $i \neq k$ then $\mathscr{I}_{\text {lock }_{i}} ; \mathscr{I}_{\text {cinc }_{k}}$ is impossible. And similarly, since

$$
\begin{aligned}
& w p(\text { unlock }, \neg(p c=1 \wedge \text { owner }=i) \\
& =(p c=1 \wedge \text { owner }=j) \Rightarrow \neg(0=1 \wedge \text { null }=i) \\
& =\text { true }
\end{aligned}
$$

it follows that unlock $_{j} ; \mathscr{I}_{\text {cinc }_{k}}$ is impossible for all values of $j$ and $k$. So

$$
\begin{aligned}
& T ; \mathscr{I}_{\text {cinc }_{k}} \\
& =\left(\text { skip } \mid \text { lock }_{k} \mid\left(\left.\right|_{i}\left(\text { unlock }_{i} ; \text { lock }_{k}\right)\right)\right) ; \mathscr{I}_{\text {cinc }_{k}}
\end{aligned}
$$

In the same way, $\mathscr{I}_{\text {cinc }_{k}} ;$ lock $_{i}$ is always impossible, and $\mathscr{I}_{\text {cinc }_{k}} ;$ unlock $_{i}$ is impossible if $i \neq k$, so

$$
\begin{aligned}
& (\mathscr{I} \backslash \mathscr{H})_{\text {cinc }_{k}} \\
& =T ; \mathscr{I}_{\text {cinc }_{k}} ; T \\
& =\left(\operatorname{skip} \backslash \text { lock }_{k} \backslash\left(\left.\right|_{i}\left(\text { unlock }_{i} ; \text { lock }_{k}\right)\right)\right) ; \mathscr{I}_{\text {cinc }_{k}} ; \\
& \quad\left(\text { skip } \backslash \text { unlock }_{k} \backslash\left(\left.\right|_{i}\left(\text { unlock }_{k} ; \text { lock }_{i}\right)\right)\right)
\end{aligned}
$$

The actions $\mathscr{I}_{c d e c_{i}}$ simplify in the same way. Also,

$$
\begin{aligned}
& (\mathscr{I} \backslash \mathscr{H})_{\text {init }} \\
& =\mathscr{I}_{\text {init }} ; T \\
& =\mathscr{I}_{\text {init }} ;\left(\operatorname{skip} \backslash\left(\left.\right|_{i} \text { lock }_{i}\right)\right)
\end{aligned}
$$

[removing impossible branches]

Finally, we make a renaming step. We use a function, $r$, which renames each cinci $_{i}$ to $\operatorname{cinc}$ and each $c d e c_{i}$ to $c d e c$. This has the effect of disjoining all the individual increment and decrement operations:

$$
\begin{aligned}
& (r(\mathscr{I} \backslash \mathscr{H}))_{\text {cinc }}=\left.\right|_{i}(\mathscr{I} \backslash \mathscr{H})_{\text {cinc }_{i}} \\
& (r(\mathscr{I} \backslash \mathscr{H}))_{\text {cdec }}=\left.\right|_{i}(\mathscr{I} \backslash \mathscr{H})_{\text {cdec }_{i}}
\end{aligned}
$$


The initialisation is unchanged:

$$
(r(\mathscr{I} \backslash \mathscr{H}))_{\text {init }}=(\mathscr{I} \backslash \mathscr{H})_{\text {init }}
$$

\subsection{The Refinement Step}

We wish the concrete variable, $v$, to have the same value as the abstract variable, $u$. This leads to the choice of abstraction relation: $R \widehat{=} u=v$. Using the refinement laws derived in [WoM90] we show that the relation $R$ satisfies

$$
R: \mathscr{P} \sqsubseteq r(\mathscr{I} \backslash \mathscr{H})
$$

That is, $R$ is a forwards simulation between the two action systems.

The values of conjugate weakest preconditions and guards used in the following proofs are calculated in Appendix A.

- The initialisation rule

For any post-condition, $\beta$, not containing the abstract variable $u$ we must prove:

$$
\begin{aligned}
& \text { 1. } \left.\left(\exists v, p c, \text { owner } \bullet \overline{w p}(r(\mathscr{I} \backslash \mathscr{H}))_{\text {init }}, \beta\right)\right) \Rightarrow \\
& \left(\exists u \bullet \overline{w p}\left(\mathscr{S}_{\text {init }}, \exists v, p c, \text { owner } \bullet u=v \wedge \beta\right)\right)
\end{aligned}
$$

Simplifying the right hand side of the implication gives:

$$
\begin{aligned}
& \text { RHS } 1 \\
& =\exists u \bullet((\exists v, p c, \text { owner } \bullet u=v \wedge \beta)[0 / u]) \\
& =(\exists v, p c, \text { owner } \bullet u=v \wedge \beta)[0 / u] \\
& =(\exists p c, \text { owner } \bullet \beta[u / v])[0 / u] \\
& =(\exists p c, \text { owner } \bullet \beta[0 / v])
\end{aligned}
$$

and the left hand side is:

$$
\begin{aligned}
& \text { LHS } 1 \\
& =(\exists v, p c, \text { owner } \bullet \beta[0 / v, 0 / p c, \text { null/owner }] \\
& \qquad(\exists i:(1 \ldots n) \bullet \beta[0 / v, 1 / p c, i / \text { owner }])) \\
& =\beta[0 / v, 0 / p c, \text { null/owner }] \\
& \quad \vee(\exists i:(1 \ldots n) \bullet \beta[0 / v, 1 / p c, i / \text { owner }])
\end{aligned}
$$

Using the One Point Rule the first disjunct is equivalent to:

$$
(\exists p c, \text { owner } \bullet p c=0 \wedge \text { owner }=\text { null } \wedge \beta[0 / v])
$$

which implies RHS 1.

The second disjunct is equivalent to:

$$
(\exists i:(1 \ldots n) \bullet(\exists p c, \text { owner } \bullet p c=1 \wedge \text { owner }=i \wedge \beta[0 / v]))
$$

and for each possible $i$, this implies RHS 1 . Since each branch of LHS 1 entails $R H S 1$ we have $L H S 1 \Rightarrow R H S 1$ as required.

- The correctness rule Again,for any post-condition, $\beta$, not containing $u$ we need to show for each action $x$ and its concrete counterpart, $c x$ :

2. $(\exists v, p c$, owner $\bullet u=v \wedge \overline{w p}(c x, \beta)) \Rightarrow \overline{w p}(x,(\exists v, p c$, owner $\bullet u=v \wedge \beta))$ 
Arguing for the operation inc:

$$
\begin{aligned}
& \text { RHS } 2 \\
& =\overline{w p}\left(\mathscr{S}_{\text {inc }}, \exists v, p c, \text { owner } \bullet u=v \wedge \beta\right) \\
& =(\exists v, p c, \text { owner } \bullet u=v \wedge \beta)[u+1 / u] \\
& =(\exists p c, \text { owner } \bullet \beta[u+1 / v]) \quad \text { [One point rule and } u \text { not free in } \beta \text { ] }
\end{aligned}
$$

Also:

\section{LHS 2}

$=(\exists v, p c, o w n e r \bullet$

$$
\begin{aligned}
& u=v \\
\wedge & ((\exists i:(1 \ldots n) \bullet \text { owner }=i \wedge \beta[2 / p c, i / \text { owner }, v+1 / v]) \vee \\
& (p c \neq 1 \wedge(\exists i:(1 \ldots n) \bullet \beta[2 / p c, i / \text { owner }, v+1 / v])) \vee \\
& (\exists i:(1 \ldots n) \bullet \beta[1 / p c, i / \text { owner }, v+1 / v]) \vee \\
& \beta[0 / p c, \text { null } / \text { owner }, v+1 / v] \\
& \\
&
\end{aligned}
$$$$
(\exists i:(1 \ldots n) \bullet \text { owner }=i \wedge \beta[2 / p c, i / o w n e r, v+1 / v]) \vee
$$$$
(p c \neq 1 \wedge(\exists i:(1 \ldots n) \bullet \beta[2 / p c, i / o w n e r, v+1 / v])) \vee
$$$$
(\exists i:(1 \ldots n) \bullet \beta[1 / p c, i / o w n e r, v+1 / v]) \vee
$$$$
\beta[0 / p c, \text { null/owner }, v+1 / v]
$$

[One Point rule and $u$ not free in $\beta$ ]

$$
\begin{gathered}
\quad \text { [One Point rule and } u \text { not free } \\
=(\exists \text { owner } \bullet(\exists i:(1 . n) \bullet \text { owner }=i \wedge \beta[2 / p c, i / \text { owner }, u+1 / v])) \vee
\end{gathered}
$$

$(\exists p c \bullet p c=0 \wedge(\exists i:(1 . n) \bullet$ owner $=i \wedge \beta[2 / p c, i /$ owner,$u+1 / v])) \vee$ $(\exists p c \bullet p c=2 \wedge(\exists i:(1 \ldots n) \bullet$ owner $=i \wedge \beta[2 / p c, i /$ owner,$u+1 / v])) \vee$ $(\exists i:(1 \ldots n) \bullet \beta[1 / p c, i / o w n e r, u+1 / v]) \vee$

$\beta[0 / p c$, null /owner, $u+1 / v]$ Removing vac. quant. and using type of $p c]$

$$
=(\exists i:(1 \ldots n) \bullet \beta[2 / p c, i / o w n e r, u+1 / v]) \vee
$$$$
(\exists i:(1 \ldots n) \bullet \beta[1 / p c, i / \text { owner }, u+1 / v]) \vee
$$$$
\beta[0 / p c, \text { null /owner, } u+1 / v]
$$

[One Point Rule]

For each possible value of $i$ each branch of $L H S 2$ implies $R H S 2$. Therefore $L H S 2 \Rightarrow R H S 2$ as required. The proof for dec follows the same pattern.

- The applicability rule

The third condition to be met is that for each action, $x$ :

3. $(\exists u \bullet u=v \wedge g d x) \Rightarrow g d c x$

which reduces to true for both the increment and the decrement operation since all guards are true.

\section{Sufficient Conditions for System with Hiding}

In the above example each hidden action alters only the auxiliary variables, $p c$ and owner. The concrete variable which plays a participating role in the 
abstraction relation, namely $v$, is not changed by any of the lock $k_{i}$, unlock actions. This pattern has also been noted in similar examples and we make use of this special case to derive sufficient conditions for $\mathscr{P} \vec{\sqsubseteq}(\mathscr{I} \backslash \mathscr{H})$. The sufficient conditions have the advantage of being easier to verify, and hence the refinement calculations for systems such as the one above become more manageable. As before, we require that repetition of the hidden actions be bounded.

The following rules use $R$ to denote the abstraction relation, $a$ for the abstract variables, and $c$ for the concrete variables. The property noted above is formalised as follows. For each hidden action, hop, for any postcondition, $\beta$ :

(I) $\left(\exists c \bullet R \wedge \overline{w p}\left(\mathscr{I}_{h o p}, \beta\right)\right) \Rightarrow(\exists c \bullet R \wedge \beta)$

The refinement conditions may then be simplified to:

$(I I) \quad\left(\exists c \bullet \overline{w p}\left(\mathscr{I}_{\text {init }}, \beta\right)\right) \Rightarrow\left(\exists a \bullet \overline{w p}\left(\mathscr{S}_{\text {init }},(\exists c \bullet R \wedge \beta)\right)\right)$

This is just the initial states rule for the concrete system without hiding.

Each unhidden action, cop, must correspond to exactly one abstract action and must obey:

(III) $\left(\exists c \bullet R \wedge \overline{w p}\left(\mathscr{I}_{c o p}, \beta\right)\right) \Rightarrow \overline{w p}(a \circ p,(\exists c \bullet R \wedge \beta))$

Again, this is simply the correctness rule for cop. The advantage of these rules is that the iteration, $T$, no longer appears, thus simplifying the calculation.

The simplified initial states and correctness rules rely on the fact that hidden operations cannot alter the "relevant" part of the concrete state. Applicability cannot be dismissed in this way since hidden actions may move the system into a state where applicability holds. However, a straight rewriting of the rule using Lemma 5 gives some simplification. For each unhidden action, cop:

$(I V) \quad\left(\exists a \bullet R \wedge g d \mathscr{S}_{a o p}\right) \Rightarrow \overline{w p}\left(T, g d \mathscr{I}_{c o p}\right)$

To show that conditions $(I)-(I V)$ are indeed sufficient we must prove the following theorem:

Theorem 1. The following are all consequences of conditions $(I)-(I V)$ :

$($ IS $) \quad\left(\exists c \bullet \overline{w p}\left(\mathscr{I}_{\text {init }} ; T, \beta\right)\right) \Rightarrow\left(\exists a \bullet \overline{w p}\left(\mathscr{S}_{\text {init }},(\exists c \bullet R \wedge \beta)\right)\right)$

(CR) $\left(\exists c \bullet R \wedge \overline{w p}\left(T ; \mathscr{I}_{c o p} ; T, \beta\right)\right) \Rightarrow \overline{w p}\left(\mathscr{S}_{\text {aop }},(\exists c \bullet R \wedge \beta)\right)$

$(A P) \quad\left(\exists a \bullet R \wedge g d \mathscr{S}_{\text {aop }}\right) \Rightarrow \mathrm{gd}(T ; \operatorname{cop} ; T)$

where $T \cong$ it $\prod_{h \in \mathscr{H}} \mathscr{I}_{h}$ ti .

Hence if $(I)-(I V)$ hold, $\mathscr{S} \overrightarrow{\underline{E}}(\mathscr{I} \backslash \mathscr{H})$.

Since the applicability is just a rewrite, there is nothing to prove for $(A P)$. To prove $(I S)$ and $(C R)$ we make use of the following lemma, a proof of which is given later.

\section{Lemma 7.}

$(\exists c \bullet R \wedge \overline{w p}(T, \beta)) \Rightarrow(\exists c \bullet R \wedge \beta)$ 
With this lemma the proofs of (IS) and (CR) follow easily.

Proof of Theorem 1.

To establish IS :

$$
\begin{aligned}
& \left(\exists c \bullet \overline{w p}\left(\mathscr{I}_{\text {init }} ; T, \beta\right)\right) \\
& =\exists c \bullet \overline{w p}\left(\mathscr{I}_{\text {init }}, \overline{w p}(T, \beta)\right) \\
& \Rightarrow\left(\exists a \bullet \overline{w p}\left(\mathscr{S}_{\text {init }},(\exists c \bullet R \wedge \overline{w p}(T, \beta))\right)\right) \\
& \Rightarrow\left(\exists a \bullet \overline{w p}\left(\mathscr{S}_{\text {init }},(\exists c \bullet R \wedge \beta)\right)\right)
\end{aligned}
$$

[Assumption]

[wp calculus]

[Using $I I]$

[Lemma 7 and monotonicity]

To establish $C R$ :

$\left(\exists c \bullet R \wedge \overline{w p}\left(T ; \mathscr{I}_{\text {cop }} ; T, \beta\right)\right)$

[Assumption]

$=\left(\exists c \bullet R \wedge \overline{w p}\left(T, \overline{w p}\left(\mathscr{I}_{c o p}, \overline{w p}(T, \beta)\right)\right)\right)$

[wp calculus]

$\Rightarrow\left(\exists c \bullet R \wedge \overline{w p}\left(\mathscr{I}_{c o p}, \overline{w p}(T, \beta)\right)\right)$

[Lemma 7]

$\Rightarrow \overline{w p}\left(\mathscr{S}_{\text {aop }},(\exists c \bullet R \wedge \overline{w p}(T, \beta))\right)$

[Using $I I I]$

$\Rightarrow \overline{w p}\left(\mathscr{S}_{\text {aop }},(\exists c \bullet R \wedge \beta)\right)$

[Lemma 7 and monotonicity]

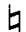

It remains to show that Lemma 7 holds. To do this we make use of the definition of $\overline{w p}$ for an iteration:

$$
\overline{w p}\left(\text { it } \prod_{h \in \mathscr{H}} \mathbf{t i}, \alpha\right)=\exists \operatorname{tr}: \mathscr{H}^{*} \bullet \overline{w p}(t r, \alpha)
$$

Using this we must show that:

$$
\left(\exists c \bullet R \wedge \exists t r: \mathscr{H}^{*} \bullet \overline{w p}(t r, \beta)\right) \Rightarrow(\exists c \bullet R \wedge \beta)
$$

Proof of Lemma 7. By induction on the length of $t r$ :

Base case: \#tr $=0$, that is $t r=\langle\rangle$ :

$$
\begin{aligned}
& \left(\exists c \bullet R \wedge\left(\exists \operatorname{tr}: \mathscr{H}^{*} \bullet \operatorname{tr}=\langle\rangle \wedge \overline{w p}(t r, \beta)\right)\right) \\
& =(\exists c \bullet R \wedge \overline{w p}(\langle\rangle, \beta)) \\
& =(\exists c \bullet R \wedge \beta)
\end{aligned}
$$

[One point rule]

[ $\overline{w p}$ of $s k i p]$

Inductive step: \#tr $>0$. Assume lemma is true for all sequences of length $n$. Then if $t r$ is a trace of length $n+1$ let head $t r=h$ so that $t r=\langle h\rangle^{-}$(tail tr) where tail $t r$ will be of length $n$.

$$
\begin{aligned}
& \left.\left(\exists c \bullet R \wedge\left(\exists \operatorname{tr}: \mathscr{H}^{*} \bullet \text { tr }=\langle h\rangle\right\urcorner(\text { tail tr }) \wedge \overline{w p}(\text { tr }, \beta)\right)\right) \\
& =(\exists c \bullet R \wedge \overline{w p}(\langle h\rangle-(\text { tail tr }), \beta)) \\
& =(\exists c \bullet R \wedge \overline{w p}(h, \overline{w p}(\text { tail tr }, \beta))) \\
& \Rightarrow(\exists c \bullet R \wedge \overline{w p}(\text { tail tr, } \beta)) \\
& \Rightarrow(\exists c \bullet R \wedge \beta)
\end{aligned}
$$

[One point rule] $[\overline{w p}$ of a trace]

[Using $I$ ] [Ind. hyp.]

七

\section{Sufficient Conditions for System with Hiding and Renaming}

Unfortunately, when a renaming step is involved the simplified rules cannot be applied immediately. The refinement conditions which must be satisfied are: 
$\left(I S^{\prime}\right) \quad\left(\exists c \bullet \overline{w p}\left(\mathscr{I}_{\text {init }} ; T, \beta\right)\right) \Rightarrow\left(\exists a \bullet \overline{w p}\left(\mathscr{S}_{\text {init }},(\exists c \bullet R \wedge \beta)\right)\right)$

$\left(C R^{\prime}\right) \quad\left(\exists c \bullet R \wedge \overline{w p}\left(\prod_{i}\left(T ; \mathscr{I}_{c o p_{i}} ; T\right), \beta\right)\right) \Rightarrow \overline{w p}\left(\mathscr{S}_{\text {aop }},(\exists c \bullet R \wedge \beta)\right)$

where the $c o p_{i}$ are the actions renamed to cop corresponding to aop.

$\left.\left(A P^{\prime}\right) \quad\left(\exists a \bullet R \wedge g d \mathscr{S}_{\text {aop }}\right) \Rightarrow g d\right|_{i}\left(T ; \mathscr{I}_{\text {cop }_{i}} ; T\right)$

Our sufficient conditions will also be slightly different. The first two remain unaltered but now we have:

$\left(I I I^{\prime}\right) \quad\left(\exists c \bullet R \wedge \overline{w p}\left(\mathscr{I}_{c o p_{i}}, \beta\right)\right) \Rightarrow \overline{w p}\left(\mathscr{S}_{\text {aop }},(\exists c \bullet R \wedge \beta)\right)$

for each $c o p_{i}$ which is renamed to cop corresponding to aop. Also:

$$
\left(I V^{\prime}\right) \quad\left(\exists a \bullet R \wedge g d \mathscr{S}_{\text {aop }}\right) \Rightarrow \overline{w p}\left(T, g d\left(\left.\right|_{i} \mathscr{I}_{\text {cop }_{i}}\right)\right)
$$

Theorem 2. If $I, I I, I I I^{\prime}$ and $I V^{\prime}$ hold then $\mathscr{S} \vec{\sqsubseteq}(\mathscr{I} \backslash \mathscr{H})$.

Proof. The initial states requirement, $I S^{\prime}$, follows exactly as before. For $C R^{\prime}$ :

$$
\begin{aligned}
& \left(\exists c \bullet R \wedge \overline{w p}\left(\left.\right|_{i}\left(T ; \mathscr{I}_{c_{c o p}} ; T\right), \beta\right)\right) \\
& =\left(\exists c \bullet R \wedge \exists i \bullet \overline{w p}\left(T ; \mathscr{I}_{c_{c o p}} ; T, \beta\right)\right) \\
& =\left(\exists i \bullet\left(\exists c \bullet R \wedge \overline{w p}\left(T ; \mathscr{I}_{c o p_{i}} ; T, \beta\right)\right)\right) \\
& \Rightarrow\left(\exists i \bullet\left(\exists c \bullet R \wedge \overline{w p}\left(\mathscr{I}_{c_{c o p}}, \overline{w p}(T, \beta)\right)\right)\right) \\
& \Rightarrow \overline{w p}\left(\mathscr{S}_{\text {aop }},(\exists c \bullet R \wedge \overline{w p}(T, \beta))\right) \\
& \Rightarrow \overline{w p}\left(\mathscr{S}_{\text {aop }},(\exists c \bullet R \wedge \beta)\right)
\end{aligned}
$$

[Defn. !]

[Reordering quantification]

[Lemma 7]

[Using $\left.I I I^{\prime}\right]$

[Lemma 7 and monotonicity]

For $A P^{\prime}$, note that RHS of $I V^{\prime}$ is:

$$
\begin{aligned}
& \overline{w p}\left(T, g d\left(\left.\right|_{i} \mathscr{I}_{c o p_{i}}\right)\right) \\
& =\overline{w p}\left(T ; \overline{w p}\left(\left.\right|_{i} \mathscr{I}_{c o p_{i}}, \text { true }\right)\right) \\
& =\overline{w p}\left(T, \exists i \bullet \overline{w p}\left(\mathscr{I}_{c o p_{i}}, \text { true }\right)\right. \\
& =\exists i \bullet \overline{w p}\left(T, \overline{w p}\left(\mathscr{I}_{c o p_{i}}, \text { true }\right)\right) \\
& =\exists i \bullet \overline{w p}\left(T, \overline{w p}\left(\mathscr{I}_{c o p_{i}}, \overline{w p}(T, \text { true })\right)\right) \\
& =\overline{w p}\left(\left.\right|_{i}\left(T ; \mathscr{I}_{c o p_{i}}\right) ; T, \text { true }\right) \\
& =\left.g d\right|_{i}\left(T ; \mathscr{I}_{c p_{i}} ; T\right)
\end{aligned}
$$

[Guard of command] [Dist. of $\vee$ for $\overline{w p}$ ] [Lemma 4]

曰

The modified conditions may now be used for our example to show that $\mathscr{S} \sqsubseteq r(\mathscr{I} \backslash \mathscr{H})$. We show that conditions $I, I I, I I I^{\prime}$ and $I V^{\prime}$ hold between $\mathscr{S}$ and $\mathscr{I}$.

$(I) \quad(\exists c \bullet R \wedge \overline{w p}(h o p, \beta)) \Rightarrow(\exists c \bullet R \wedge \beta)$ 
For each lock $_{i}$ LHS is:

$$
\begin{aligned}
& \left(\exists v, p v, \text { owner } \bullet u=v \wedge \overline{w p}\left(\text { lock }_{i}, \beta\right)\right) \\
& =(\exists v, p c, \text { owner } \bullet u=v \wedge p c=0 \wedge \text { owner = null } \wedge \beta[1 / p c, i / \text { owner }]) \\
& =(\exists v \bullet u=v \wedge \beta[1 / p c, i / \text { owner }]) \\
& \Rightarrow(\exists v, p c, \text { owner } \bullet u=v \wedge \beta)
\end{aligned}
$$

and similarly for unlock $_{i}$.

(II) $\left(\exists c \bullet \overline{w p}\left(\mathscr{I}_{\text {init }}, \beta\right)\right) \Rightarrow\left(\exists a \bullet \overline{w p}\left(\mathscr{S}_{\text {init }},(\exists c \bullet R \wedge \beta)\right)\right)$

$L H S=\left(\exists v, p c\right.$, owner $\left.\bullet \overline{w p}\left(\mathscr{I}_{\text {init }}, \beta\right)\right)$

$=\beta[0 / v, 0 / p c$, null /owner $]$

$$
\begin{aligned}
& R H S=(\exists u \bullet(\exists v, p c, \text { owner } \bullet u=v \wedge \beta)[0 / u]) \\
& =(\exists p c, \text { owner } \bullet \beta[0 / v])
\end{aligned}
$$

And so $L H S \Rightarrow R H S$.

$$
\left(I I I^{\prime}\right) \quad\left(\exists c \bullet R \wedge \overline{w p}\left(\mathscr{S}_{\text {cop }_{i}}, \beta\right)\right) \Rightarrow \overline{w p}\left(\mathscr{S}_{\text {aop }},(\exists c \bullet R \wedge \beta)\right)
$$

For $\operatorname{cinc}_{i}$ :

$$
\begin{aligned}
& \text { LHS }=\left(\exists v, p v, \text { owner } \bullet u=v \wedge \overline{w p}\left(\text { cinc }_{i}, \beta\right)\right) \\
& =(\exists v, p v, \text { owner } \bullet u=v \wedge p c=1 \wedge \text { owner }=i \wedge \beta[2 / p c, v+1 / v]) \\
& =(\exists v \bullet u=v \wedge \beta[2 / p c, v+1 / v, i / \text { owner }] \\
& =\beta[2 / p c, u+1 / v, i / \text { owner }] \quad \quad[u \text { not free in } \beta] \\
& \text { RHS }=(\exists v, p c, \text { owner } \bullet u=v \wedge \beta)[u+1 / u] \\
& =(\exists p c, \text { owner } \bullet \beta[u+1 / v])
\end{aligned}
$$

Hence $L H S \Rightarrow R H S$, and similarly for each $c d e c_{i}$.

$$
\left(I V^{\prime}\right) \quad\left(\exists a \bullet R \wedge g d \mathscr{P}_{a o p}\right) \Rightarrow \overline{w p}\left(T, g d\left(\left.\right|_{i} \mathscr{I}_{c o p_{i}}\right)\right)
$$

The LHS is equivalent to true and since $g d\left(\prod_{i} \mathscr{I}_{c i n c_{i}}\right)=p c=1$ :

$$
\begin{aligned}
& \text { RHS }=\overline{w p}(T, p c=1) \\
& =p c=1 \vee(p c=0 \wedge \text { owner }=\text { null }) \vee p c=2 \\
& =\text { true }
\end{aligned}
$$

[Using invariant and type of $p c$ ]

Thus all four conditions are easily verified, confirming the results achieved at greater length in Section 7.

\section{Related Work}

The material presented here is closely related to a large body of work in the area of action systems and refinement of state-based parallel programs. State-based 
concurrency and refinement in the failures model of CSP were investigated by He Jifeng [Jif89] and Josephs [Jos88]. Both use a relational approach in which actions are relations between states. He Jifeng also defines divergences, introducing a bottom state, $\perp$, for this purpose.

Morgan [Mor90] makes a similar link, but here the correspondence between state- and event-based views is given using weakest preconditions. CSP traces, failures and divergences are defined by weakest precondition expressions over action systems. This approach has the advantage of treating divergence uniformly without requiring a special non-terminating state. Further work in this model was carried out in [WoM90], providing a set of rules for action system refinement. These rules were shown to be correct with respect to CSP refinement.

The development of action system theory can be traced through many papers by Back et al., such as [BKS83], [BaS89], [BvW89], [Bac89]. The earlier of these describe action systems and provide a method (known as superposition) for their stepwise refinement in a temporal logic framework. The more recent describe how refinement may be done in the refinement calculus. Superposition allows for refinement between action systems where the concrete system may have additional variables and actions. The conditions to be satisfied, as set out in Theorem 4 of [BaS90], are very similar to those of our Theorem 1. The alteration in atomicity is allowed for by permitting stuttering actions in the concrete system. These cannot change any state component common to the abstract system and are thus similar to the actions allowed in $\mathscr{H}$ of our Theorem 1. Stuttering and stuttering-equivalence were introduced by Lamport [Lam89] and used [AbL88] for the implementation of concurrent systems specified by the transition-axiom method. Again, this approach is based in temporal logic.

Our work follows that of [Mor90], [WoM90],with action systems given meaning in the CSP failures-divergence model. Hiding and renaming operators, defined to correspond to their CSP counterparts, are used to account for alteration of atomicity within action system refinement. The case study provides an example of their use. Here, the purpose of the silent operations was to allow the locking and unlocking of a resource because the target language does not have atomic actions for the operations described in the specification. It is necessary to show that the iteration of hidden actions cannot lead to divergence in the system. This was achieved in this case by using a program control variable to specify the order in which actions may be performed.

The results of Sections 8 and 9 provide sufficient conditions for proving refinements in which the concrete system uses hiding and renaming. These are useful as they simplify the calculation required. There is no need to calculate explicitly the resulting concrete system, and many of the troublesome iterations are removed from the proof obligations.

Further work in this area is being carried out by Butler [BuM91], [But93]. Apart from his work in providing a link between action systems and the infinite traces model of CSP, he has also developed parallel composition for action systems in accordance with this interpretation.

Perhaps it remains to be seen which approach is most suitable for which applications, with some trade-off between general applicability and simplicity of reasoning. Certainly the correspondence between action systems and CSP seems attractive, with action systems given meaning in terms of the already well-defined notion of CSP processes. 


\section{Acknowledgements}

We thank Michael Butler, Peter Lupton and Carroll Morgan for their ideas, discussion and comment on this work. We also thank IBM United Kingdom Laboratories Ltd. for their support and collaboration.

\section{Appendix A - Conjugate Weakest Preconditions and Guards}

We first calculate the $\overline{w p}$ of $T$ as defined in Section 7.1.

$$
\begin{array}{cc}
\overline{w p}(T, \alpha) & \\
=\overline{w p}\left(\operatorname{skip} \backslash\left(\left.\right|_{i} \operatorname{lock}_{i}\right) \mid\left(\left.\right|_{i} \text { unlock }_{i}\right) \mid\left(\left.\right|_{i, j}\left(\text { unlock }_{i} ; \text { lock }_{j}\right)\right), \alpha\right) & \text { [Lemma 6] } \\
=\overline{w p}(\operatorname{skip}, \alpha) \vee \overline{w p}\left(\left.\right|_{i} \operatorname{lock}_{i}, \alpha\right) \vee \overline{w p}\left(\left.\right|_{i} \text { unlock }_{i}, \alpha\right) & \\
\vee \overline{w p}\left(\prod_{i, j}\left(\text { unlock }_{i} ; \text { lock }_{j}\right), \alpha\right) & {[\overline{w p}]}
\end{array}
$$

The first disjunct simplifies to $\alpha$. Next:

$$
\begin{array}{lr}
\overline{w p}\left(\left.\right|_{i} l o c k_{i}, \alpha\right) & \\
=\left(\exists i:(1 \ldots n) \bullet \overline{w p}\left(\text { lock }_{i}, \alpha\right)\right) & {[\overline{w p} \mid]} \\
=(\exists i:(1 \ldots n) \bullet p c=0 \wedge \text { owner }=\text { null } \wedge \alpha[1 / p c, i / o w n e r]) & \text { [defn.] } \\
=p c=0 \wedge \text { owner }=n u l l \wedge(\exists i:(1 \ldots n) \bullet \alpha[1 / p c, i / \text { owner] }) & \text { [pred. calc.] } \\
=p c=0 \wedge(\exists i:(1 . n) \bullet \alpha[1 / p c, i / \text { owner }]) & \text { [invariant] }
\end{array}
$$

The third disjunct simplifies similarly:

$$
\begin{aligned}
& \overline{w p}\left(\left.\right|_{i} \text { unlock }_{i}, \alpha\right) \\
& =\left(\exists i:(1 \ldots n) \bullet \overline{w p}\left(\text { unlock }_{i}, \alpha\right)\right) \\
& =(\exists i:(1 \ldots n) \bullet p c=2 \wedge \text { owner }=i \wedge \alpha[0 / p c, \text { null/owner }]) \\
& =p c=2 \wedge \text { owner }=i \wedge(\exists i:(1 \ldots n) \bullet \alpha[0 / p c, \text { null/owner }]) \\
& =p c=2 \wedge \alpha[0 / p c, \text { null/owner }]
\end{aligned}
$$

And for the fourth branch:

$$
\begin{aligned}
& \overline{w p}\left(\prod_{i, j}\left(\text { unlock }_{i} ; \text { lock }_{j}\right), \alpha\right) \\
&=\left(\exists i, j:(1 \ldots n) \bullet \overline{w p}\left(\text { unlock }_{i} ; \text { lock }_{j}, \alpha\right)\right) \\
&=\left(\exists i, j:(1 \ldots n) \bullet \overline{w p}\left(\text { unlock }_{i}, \overline{w p}\left(\text { lock }_{j}, \alpha\right)\right)\right) \\
&=(\exists i, j:(1 \ldots n) \bullet \\
&\quad \overline{w p}(\text { unlock }, p c=0 \wedge \text { owner }=\text { null } \wedge \alpha[1 / p c, j / \text { owner }])) \\
&=(\exists i, j:(1 \ldots n) \bullet \\
& \quad p c=2 \wedge \text { owner }=i \wedge \\
& \quad(p c=0 \wedge \text { owner }=\text { null } \wedge \alpha[1 / \text { calc] } \\
& \text { [defn.] }
\end{aligned}
$$




$$
\begin{array}{lr}
=(\exists i, j:(1 \ldots n) \bullet p c=2 \wedge \text { owner }=i \wedge \alpha[1 / p c, j / \text { owner] }) & \text { [applying sub.] } \\
=p c=2 \wedge(\exists j:(1 \ldots n) \bullet \alpha[1 / p c, j / \text { owner }]) & \text { [pred. calc.] }
\end{array}
$$

Putting these together gives:

$$
\begin{aligned}
\overline{w p}(T, \alpha)=\alpha \vee(p c=0 & \wedge(\exists i:(1 \ldots n) \bullet \alpha[1 / p c, i / \text { owner }])) \vee \\
(p c=2 & \wedge \alpha[0 / p c, \text { null/owner }]) \vee \\
(p c=2 & \wedge(\exists i:(1 \ldots n) \bullet \alpha[1 / p c, i / \text { owner }]))
\end{aligned}
$$

Using the type of $p c$ this simplifies to:

$$
\begin{gathered}
\overline{w p}(T, \alpha)=\alpha \vee(p c \neq 1 \wedge(\exists i: \text { owner }:(1 \ldots n) \bullet \alpha[1 / p c, i / \text { owner }])) \vee \\
(p c=2 \wedge \alpha[0 / p c, \text { null /owner }])
\end{gathered}
$$

The above result is used in the following calculations necessary for the refinement proof.

$$
\begin{aligned}
& \overline{w p}\left(\mathscr{S}_{\text {init }}, \alpha\right)=\alpha[0 / u] \\
& \overline{w p}\left(\mathscr{S}_{i n c}, \alpha\right)=\alpha[u+1 / u] \\
& \overline{w p}\left((\mathscr{I} \backslash \mathscr{H})_{\text {init }}, \alpha\right) \\
& =\overline{w p}\left((\mathscr{I} \backslash \mathscr{H})_{\text {init }} ; T, \alpha\right) \\
& =\overline{w p}\left((\mathscr{I} \backslash \mathscr{H})_{\text {init }}, \overline{w p}(T, \alpha)\right) \\
& =(\overline{w p}(T, \alpha))[0 / v, 0 / p c, \text { null /owner }] \\
& =\alpha[0 / v, 0 / p c, \text { null } / \text { owner }] \vee(\exists i:(1 \ldots n) \bullet \alpha[0 / v, 1 / p c, i / o w n e r]) \\
& \overline{w p}\left((\mathscr{I} \backslash \mathscr{H})_{\text {cinc }_{i}}, \alpha\right) \\
& =\overline{w p}\left(T ; \mathscr{I}_{\text {cinc }_{i}} ; T, \alpha\right) \\
& =\overline{w p}\left(T, \overline{w p}\left(\mathscr{I}_{c i n c_{i}}, \overline{w p}(T, \alpha)\right)\right) \\
& =\overline{w p}(T, p c=1 \wedge \text { owner }=i \wedge(\overline{w p}(T, \alpha))[2 / p c, v+1 / v]) \\
& =\overline{w p}(T, p c=1 \wedge \text { owner }=i \wedge \\
& (\alpha \vee(p c \neq 1 \wedge(\exists j:(1 \ldots n) \bullet \alpha[1 / p c, j / \text { owner }])) \\
& \vee(p c=2 \wedge \alpha[0 / p c, \text { null /owner }]))[2 / p c, v+1 / v]) \\
& =\overline{w p}(T, p c=1 \wedge \text { owner }=i \wedge \\
& (\alpha[2 / p c] \vee(\exists j:(1 \ldots n) \bullet \alpha[1 / p c, j / \text { owner }]) \\
& \vee \propto[0 / p c, \text { null /owner }])[v+1 / v]) \\
& =(p c=1 \wedge \text { owner }=i \wedge(\alpha[2 / p c] \vee(\exists j:(1 \ldots n) \bullet \alpha[1 / p c, j / \text { owner }]) \\
& \vee \propto[0 / p c, \text { null/owner }])[v+1 / v]) \\
& \checkmark \\
& (p c \neq 1 \wedge(\exists k:(1 . n) \bullet k=i \wedge(\alpha[2 / p c, k / \text { owner }] \\
& \vee(\exists j:(1 \ldots n) \bullet \alpha[1 / p c, j / \text { owner }]) \\
& \vee \propto[0 / p c, \text { null /owner }])[v+1 / v])) \\
& =(p c=1 \wedge \text { owner }=i \wedge(\alpha[2 / p c] \vee(\exists j:(1 \ldots n) \bullet \alpha[1 / p c, j / \text { owner }]) \\
& \vee \alpha[0 / p c, \text { null /owner }])[v+1 / v]) \\
& \checkmark \\
& (p c \neq 1 \wedge(\alpha[2 / p c, i / o w n e r] \vee(\exists j:(1 \ldots n) \bullet \alpha[1 / p c, j / \text { owner }]) \\
& \text { - } \vee \alpha[0 / p c, \text { null/owner }])[v+1 / v])
\end{aligned}
$$




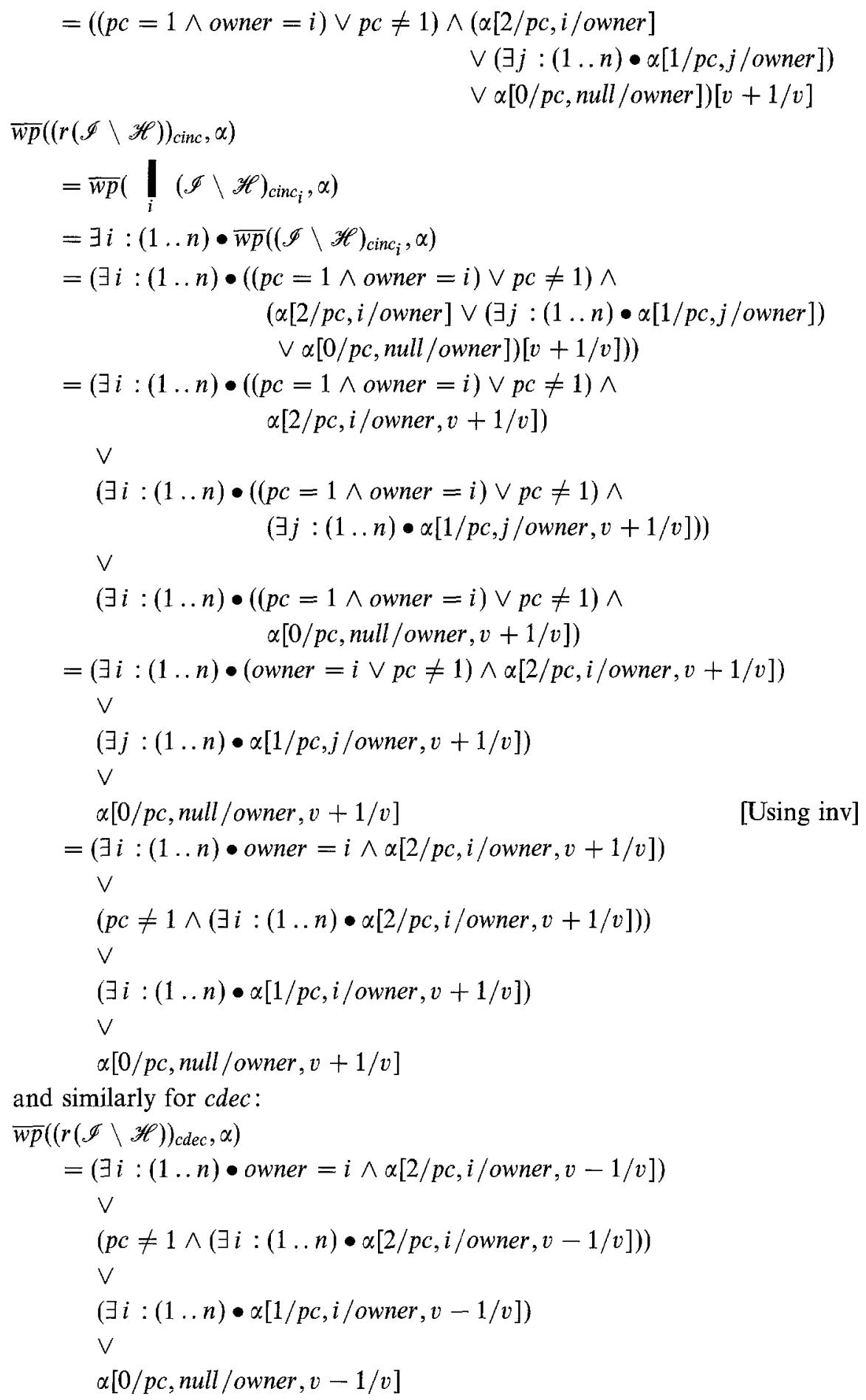


Also, for the guards of the concrete actions:

$$
\begin{aligned}
& \overline{w p}\left((r(\mathscr{I} \backslash \mathscr{H}))_{\text {cinc }}, \text { true }\right)=\text { true } \\
& \overline{w p}\left((r(\mathscr{I} \backslash \mathscr{H}))_{\text {cdec }}, \text { true }\right)=\text { true }
\end{aligned}
$$

\section{References}

[AbL88] Abadi, M. and Lamport, L.: The existence of refinement mappings. Proc 3rd IEEE Symposium on LICS, Edinburgh 1988.

[Bac89] Back, R-J. R.: Refinement calculus II: Parallel and reactive programs. Proc REX Workshop on Stepwise Refinement of Distributed Systems: Models, Formalisms, Correctness, J.W. de Bakker, W.-P. de Roever and G. Rozenburg (eds), Springer-Verlag, LNCS 430, 1989.

[BaS89] Back, R-J. R. and Sere, K.: Refinement of action systems. Mathematics of Program Construction, Springer-Verlag,LNCS 375,1989.

[BaS90] Back, R-J. R. and Sere, K.: Deriving an Occam Implementation of Action Systems. Proc 3rd Refinement Workshop, Carroll Morgan \& J.C.P. Woodcock (eds), Springer-Verlag, 1990.

[BKS83] Back, R-J. R. and Kurki-Suonio, R.: Decentralization of process nets with centralized control. Proc 2nd Annual Symposium on Principles of Distributed Computing, Montreal 1983.

[BuM91] Butler, M. and Morgan, C.: Action Systems, Unbounded Nondeterminism and Infinite Traces. Oxford University Computing Laboratory, Programming Research Group 1991.

[But93] Butler, M.: DPhil thesis. Oxford University Computing Laboratory, Programming Research Group 1993.

[BvW89] Back, R-J. R. and von Wright, J.: Refinement calculus I: Sequential nondeterministic programs. Proc REX Workshop on Stepwise Refinement of Distributed Systems: Models, Formalisms, Correctness, J.W. de Bakker, W.-P. de Roever and G. Rozenburg (eds), Springer-Verlag, LNCS 430, 1989.

[Dij76] Dijkstra, E. W.: A Discipline of Programming. Prentice-Hall International, 1976.

[Hoa85] Hoare, C. A. R.: Communicating Sequential Processes. Prentice-Hall International, 1985.

[HoK91] Houston, I. and King, S.: CICS project report: experiences and results from the use of $\mathrm{Z}$ in IBM. Proceedings of the VDM-91 Symposium, Springer-Verlag,LNCS 551,1991.

[Jif89] Jifeng, H.: Process Refinement. (ed) The Theory and Practice of Refinement, J. McDermid (ed), Butterworths, 1989.

[Jos88] Josephs, M. B.: A state-based approach to communicating sequential processes. Distributed Computing,3,9-18, 1988.

[Lam89] Lamport, L.: A simple approach to specifying concurrent systems. Communications of the ACM, 32(1):32-45, January 1989.

[Mor90] Morgan, C.: Of $w p$ and CSP. Beauty is our Business, A.J.M. van Gasteren, W.H.J. Feijen, D. Gries, and J. Misra (eds), Springer-Verlag,1990.

[Ros88] Roscoe, A. W.: Unbounded nondeterminism in CSP. Technical Report PRG-67, Programming Research Group, Oxford University, July 1988.

[Spi89] Spivey, J. M.: The $\boldsymbol{Z}$ Notation: A Reference Manual. Prentice-Hall International, 1989.

[WoM90] Woodcock, J. C. P. and Morgan, C.: Refinement of State-Based Concurrent Systems, Proceedings of the VDM-90 Conference, Springer-Verlag,LNCS 428,1990.

Received October 1991, initially handled by J. van de Snepscheut

Finally accepted in revised form July 1994 by C. B. Jones 